\title{
On the Capacity and Scalability of Wireless Mesh Networks
}

\author{
Yonghui Chen \\ Dept. of Electronics and Information Engineering of HUST \\ $\mathcal{E}$ Wuhan National Laboratory for Optoelectronic \\ Hubei University of Technology
}

\section{Introduction}

In practicable multi-user wireless networks, the communication should do among any nodes over the coverage. Since the nature of wireless channel is fading and share, the interferences and the collision becomes unable to avoid. It is difficult to balance reuse and interference while communications, location and mobility of each node are almost random. Considerate the cost, a practicable multi-user networking should have to be interference limited. Even though the Shannon capacity limitation for the single channel could be achieved by Turbo Coding(Berrou, Glavieux et al. 1993) or the MIMO (G.J.Foschini 1996) (E.Telatar 1999) technologies. In the other words, the capacity is always determined by the SIR or SINR. The flourishing cellular system and IEEE 802.11 networks are typical interference limited systems also.

It is well known that the capacity on networks is related to the networking architecture. For some type central controlled infrastructure system, e.g. a single cellular cell with FDMA CDMA or TDMA, the capacity upper bound is often assured. But the capacity on common wireless networks is still illegible, even including the multi-cell cellular system (T.M.Cover \& J.A.Thomas 2006).

Without regard to the architecture and the access mode, the abstract capacity of a wireless system could be classified in two types:

- For the typical inference limited systems, the capacity of each node should be (Gupta \& Kumar 2000; Kumar 2003) :

$$
C_{\text {node }}=\theta(1 / \sqrt{K}) \text { or } C_{\text {node }}=\theta(1 / \sqrt{K \log K})
$$

- For a X networking, in which each node has useful information to all the other nodes, the capacity of each node should be (Cadambe \& Jafar 2007; Cadambe \& Jafar 2008; Cadambe \& Jafar 2009) :

$$
C_{\text {node }}(S N R)=\theta(1)
$$

Where $\theta(\bullet)$ indicates the relation of equivalence; $K$ is the number of nodes. Formula (1) shows that the capacity of a node is inverse ratio to the $\sqrt{K}$ or $\sqrt{K \log K}$. In the other words, the capacity is decided by the SINR or SIR. Formula (2) shows the capacity could be 
unattached to the number of the nodes in the system. In the other words, if all the signal power could be taken as useful mutual information other than interference, the capacity should be limited by the SNR other than used SINR or SIR. In fact, formula (2) assumed the networking as an ideal cooperative MIMO system.

For a $\mathrm{X}$ networking with $\mathrm{S}$ source nodes, $\mathrm{D}$ destination nodes and $\mathrm{R}$ relay nodes, say each nodes has full-duplex ability, the upper bound of capacity should be (Cadambe \& Jafar 2007; Cadambe \& Jafar 2008; Cadambe \& Jafar 2009):

$$
C_{\text {node }}(S N R)=\theta[S D / K(S+D-1)]
$$

This means the capacity on multi-hop systems should be less than the one hop system. However, Wireless mesh network (WMN) has been regarded as an alternative technology for last-mile broadband access, as in fig 1.

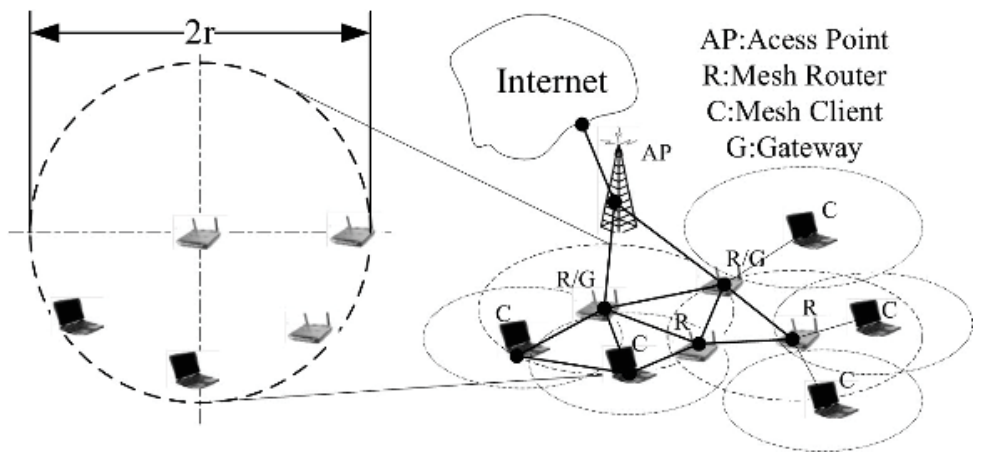

Fig. 1. A typical application of WMN. Typical nodes in WMN are Mesh Routers and Mesh Clients. Mesh clients form ad hoc sub-networks. Mesh routers form the mesh backbone for the mesh clients. Each node in WMN could act as a relay, forwarding traffic generated by other nodes.

Most industrial standards groups are actively specifying WMN, e.g. IEEE 802.11/802.15/802.16 and 3GPP LTE. For the combination of infrastructure and selforganized networking brings many advantages such as low up-front cost, robustness and reliable service coverage, etc. While WMN can be built upon existing technologies, spot test proved that the performance is still far below expectations. One of the most challenge problem is the avaliable capacity based practicable rule(Goldsmith 2005). Gennerally, similar capacity problems are slided over by simplier resource redundance(Akyildiz \& Xudong 2005). In this paper, the Asymptotic Capacity on WMN will be talked about, mainly based on the former paper(Chen, Zhu et al. 2008).

\section{Characteristic of multi-hops wireless mesh networking}

\subsection{The optimal architecture of multi-hop networking is still illegible}

The shared channel leads to hidden terminals and exposed terminals(Gallager 1985). It is a series of handshake signals that could resolve these problems to a certain extent(Karn Sept.1990; Bharghavan, Demers et al. Aug. 1994). In balance, the capacity has to bound the successful throughput on collision-free transmissions as in fig 2. 
Due to lack of any centralized controls and possible node mobility, it is hard to transplant the mature techniques from the central controlled or wired networking to the multi-hops wireless networking with high resource efficiency, which used to rely on the networking infrastructure (Basagni, Turgut et al. 2001) (Haartsen 2000) (Akyildiz \& Xudong 2005; Nandiraju, Nandiraju et al. 2007). And the medium access scheme is also a challenge for the self-organized neworking(Gupta \& Kumar 2000): Use of TDMA or dynamic assignment of frequency bands is complex since there is no centralized control; FDMA is inefficient in dense networks; CDMA is difficult to implement due to the inorganization networking. It is hard to keep track of the frequency-hopping patterns and/or spreading codes for all the nodes. the optimal architecture to the multi-hop systems is still illegible (Goldsmith 2005).

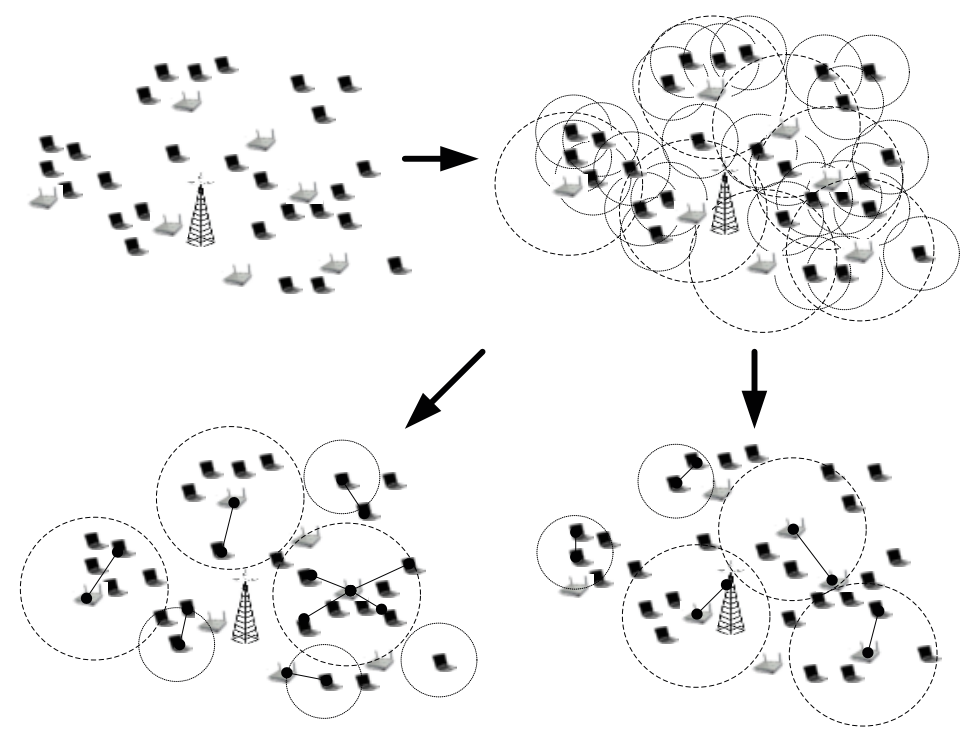

Fig. 2. Whether one hop networking or multiple hop netowrkig, practicable wireless communication system should be based on available resource reuse. The communication should be hop hy hop.

\subsection{Power Gains of ideal multi-hop link}

With an ideal linearity multi-hop chain, obviously the shorter propagating distance the more power gains. Say $\sigma_{n}^{2}$ is the noise variance, $P$ is the transfer power of each node, $K \bullet d^{-\gamma}, \gamma \geq 2$ is the path loss, where $K$ is constant, $d$ is the whole distance and $\gamma$ is path loss facter. Thus the end to end frequency normalized capacity is:

$$
C=\log \left[1+\frac{K \bullet P}{\sigma_{n}^{2} d^{\gamma}}\right]
$$

Say $N_{\text {hop }}$ is the number of hops. $d_{i}$ is the distance of the i-th hop, obviously $d \leq \sum_{i=1}^{N_{\text {hop }}} d_{i}$. Say $d_{\max }=\max \left\{d_{i}\right\}$, thus: 


$$
C=\log \left[1+\frac{K P}{\sigma_{n}^{2} d_{i}^{\gamma}}\right] \geq \log \left[1+\frac{K P}{\sigma_{n}^{2} d_{\max }^{\gamma}}\right]
$$

Since $N_{\text {hop }}$ times relay, the SNR gain of $N_{\text {hop }}$ systems is:

$$
\left[\frac{1}{N_{\text {hop }}}\left(\frac{K P}{\sigma_{n}^{2} d_{\max }^{\gamma}}\right) \div\left(\frac{K P}{\sigma_{n}^{2} d^{\gamma}}\right)\right]_{\mathrm{dB}}=10 \lg \left(\frac{1}{N_{\text {hop }}}\left(\frac{d}{d_{\max }}\right)^{\gamma}\right)
$$

Whrere $N_{\text {hop }} \geq 1, \gamma \geq 2$. If $d / d_{\max }=N_{\text {hop }}$, the gain is $10(\gamma-1) \lg \left(N_{\text {hop }}\right) \mathrm{dB}$.

\subsection{Constraints of multi-hop systems}

Even if the multi-hop link is ideal, increasing with $N_{\text {hop }}$, the link need at least $N_{\text {hop }}$ times transfer cost, e.g. the delay will be direct ratio with $N_{\text {hop }}$. Say the maximum capacity of each hop is constant 1. As a) in fig 3, despite of the hidden and exposed terminals problems, the last hop near the destination node is the bottleneck determining the capacity, with the fairness scheme. It is obviously that capacity per-node is $1 / N_{\text {hop }}$. As b) in fig 3 , with virtual circuit mode, each hop relay has the same payload, thus there is only one efficient payload from the source to the destination, capacity per-node also is $1 / N_{\text {hop }}$. In balance either absolute fairness scheme or monopolization mode, the utmost throughput per-node is $1 / N_{\text {hop }}$.

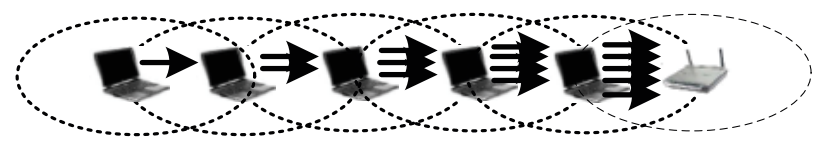

a) Relaying based on absolute fairness scheme

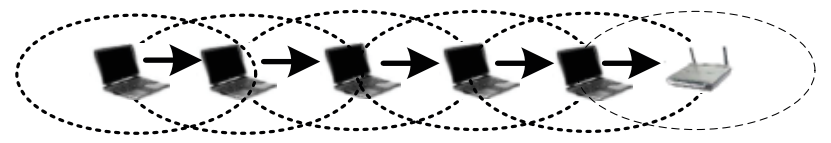

b) Relaying based on virtual circuit mode

Fig. 3. Constraints of multi-hop systems

Due to the shared channels, the hidden and exposed terminals problems are inevitable in multi-hop fashion communication. By using multiple channels/radios, or the other methods to decreases the delay, but the transfer do not truly enhance the resource utilization efficiency.

Considerate access competition, say each hop is independent and has probability $p_{c}$ to success, if the transfer time is limited to 1 , thus the access probability of a $N_{\text {hop }}$ hops chain is:

$$
p_{S}=p_{c}^{N_{\text {hop }}}
$$

If without limitation of retransfer times, the access probability is 1 : 


$$
1=\prod_{i=1}^{N_{\text {hop }}} \sum_{i=0}^{\infty} p_{c}\left(1-P_{c}\right)^{i}
$$

Say the delay of each competiction time is $T$, the expection of total delay is:

$$
\begin{aligned}
E\left(T_{D}\right) & =T \bullet \sum_{j=1}^{N_{\text {hop }}} \sum_{i=0}^{\infty}(1+i) \bullet P_{c} \bullet\left(1-P_{c}\right)^{i} \\
& =T \bullet N_{P} / P_{c}
\end{aligned}
$$

Take the average retransfer times regarded as:

$$
N_{\text {hop }}^{\prime}=N_{\text {hop }} / P_{c}
$$

Thus the actual spectrum efficiency is:

$$
1 / N_{\text {hop }}^{\prime}=P_{c} / N_{\text {hop }}
$$

\subsection{Mobility is dilemma}

There are many research focus on mobility of mesh nodes (Gupta \& Kumar 2000; Jangeun \& Sichitiu 2003; Tavli 2006). It could proved that the mobility of nodes, either random or bounded, could improve the capacity of multi-hop wireless networks by deducing the hops between the source-destination chains, as in fig 4(Grossglauser \& Tse 2002; Diggavi, Grossglauser et al. 2005). But Mobility is obviously a dilemma problem. Because too much mobility limited the capacity of multi-hop wireless networks, if considerate the cost (Jafar 2005)

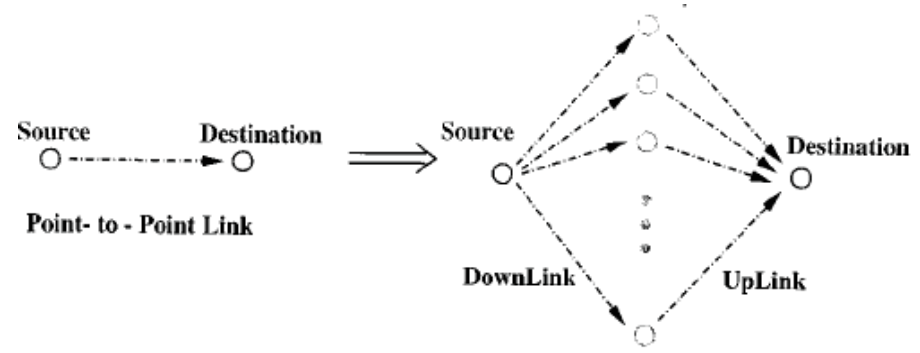

Fig. 4. Say the mobility is random, the mobile relay node has enough storage, the node as in a certain area or move along a fix path. The message could be transfered to the destination in probability with less hops.

\section{Probability model on random multi-access multi-hop system}

\subsection{Assumption}

- Say $R$ is the radium of wireless network coverage, and $N$ is the number of nodes on the area, thus the node density is $\rho_{D}=N / \pi R^{2}$;

- $\quad$ Considerate the path fading, Say each node has the same coverage, $r$ is the radium; 
- $\quad \omega$ dentes the transfer capability during a transfer period.Say $\omega$ is the same for each node;

- Say the location of the nodes is symmetrical if the scale is lager than $2(1+2 \Delta) r$, and the locations is random if the scale is smaller than $2(1+2 \Delta) r$. Where $\Delta$ is the interference limitation facter. Thus the number of node in a node cell, $n_{\text {cell }}$, is random.

- $\quad$ Say each node learn the transfer direction and send the message to these direction, and there is ideal whole networking synchronization, thus if one node get the channel at a competition slot, the transfer will be success during the next slot. In the other words, if each node has the same sending probability and similar payload, each hop of the multihop chain could be model as independent.

\subsection{Traffic model}

The networks traffics could mainly be classified in three styles: unicast traffic (Gupta \& Kumar 2000), multicast traffic (Tavli 2006) and backhaul traffic(Jangeun \& Sichitiu 2003). Note that the capacity of broadcast traffics and the backhaul traffics are equivalent in (Jangeun \& Sichitiu 2003; Tavli 2006). The collision domain of backhaul traffics obviously happen to the nodes near the gateway, while the broadcast traffics are transferring the same payload. In any case, each transmission traffics must be hop-by-hop even if the node has possible mobility as in (Grossglauser \& Tse 2002; Diggavi, Grossglauser et al. 2005). This means that the efficiency of a multi-hop chain is decide by the hops, at least partially. And each node in the chain(s) could carry no more than $\omega / N_{\text {hop }}$ efficient payload. For the different traffics there are different equivalent hops.

- For unicast traffics, Take $N_{\text {hop }}$ as the sum hops in the multi-hop chain;

- For broadcast traffic, Take $N_{\text {hop }}$ as the sum hops of all the broadcast source-termination pairs;

- $\quad$ For multicast traffic, Take $N_{\text {hop }}$ as the sum hops of each multi-hop chain.

\subsection{The connectivity model}

The model is similar to the connectivity model in (Miorando \& Granelli 2007). Model the spatial positions of each nodes as a Poisson distribution as in (Miorando \& Granelli 2007) (Takagi \& Kleinrock 1984). We have assumed each node could get the neighbors positions information, thus each node transmits its traffic directly to the very neighbor and the probability has $\mathrm{k}$ forward node is:

$$
p\left(k ; \lambda=n_{f}\right)=\frac{e^{-n_{f}} n_{f}^{k}}{k !}
$$

For Omni-antenna, take $n_{f}=n_{\text {cell }} / 2$ as in [20]. For smart antenna technology, $n_{f}$ could be a weighted $n_{\text {cell }}$. Denote $\mathrm{E}($.$) as the mathematical expectation. In any case:$

$$
n_{f}=c_{1} E\left(n_{\text {cell }}\right), \quad c_{1} \in[0,1]
$$

For simplify the analysis, normalized $\rho$ as $n_{\text {cell }} / N$, thus

$$
\rho=E\left(n_{\text {cell }}\right) / N=\left(\rho_{D} \pi r^{2}\right) /\left(\rho_{D} \pi R^{2}\right)=(r / R)^{2}
$$


(13) can be rewrite (15) as:

$$
n_{f}=c_{1} \rho N, \quad c_{1} \in(0,1], \rho \in(0,1]
$$

By the model, the probability a node has no avaliable next hop relay or terminal node is:

$$
\begin{aligned}
& p_{\text {isol }}=P\left(k=0 ; n_{f}\right)=e^{-n_{f}} \\
& E\left(p_{\text {isol }}\right)=e^{-E\left(n_{f}\right)}=e^{-c_{1} \rho N}
\end{aligned}
$$

\subsection{The access model}

Even if a node has available relay, it does not mean the node could always transmit the message successfully. With fading and shared wireless channels, a competitive access should be necessarily either in fully self-organized sytems or partially self-organized system. Therefore, a node with sending probability a does not mean has the accessable probability $a$. Assumed that the whole networking is synchronous as IEEE 802.11 DCF (Pham, Pham et al. 2005; Samhat, Samhat et al. 2006; Khayyat, Gebali et al. 2007), and the nodes have the same probability to send. Thus the collision of each-hop is independent and has the same probability distribution. In any case, assumed each node could send the message successfully with probability $u$, while the sending probability is $a$, with some backoff algorithm. Thus the successfully probability of a $n$ hop chain is:

$$
p_{f}=u^{n}
$$

The mathematical expectation of $p_{f}$ is:

$$
E\left(p_{f}\right)=\sum_{k=1}^{\rho_{D} \pi(r+\Delta)^{2}} \frac{e^{-n_{\text {cell }}} n_{\text {cell }}{ }^{k}}{k !}(u)^{k}
$$

Where take $\lambda=n_{\text {cell }}=\rho_{D} \pi r^{2}=\rho \mathrm{N}$. Considerate the collision probability will increase rapidly with the density of the nodes, in this case $u \bullet n_{\text {cell }}$ will be smaller.

$$
E\left(p_{f}\right) \approx e^{-\rho \bullet N}\left(e^{u \bullet \rho \bullet N}-1\right)
$$

while $\rho_{D} \pi(r+\Delta)^{2} \geq 5$

\section{Asymptotic capacity model on multi-hop systems}

\subsection{The capacity model}

Say the traffic over the $j$-th sub-channel has $h_{i, j}$ hops. Derived from the throughput definition in (Gupta \& Kumar 2000), the average capacity of each node can be defined as:

$$
C_{X(i)}=\sum_{j}^{N_{c h}(i)}\left\{\prod_{h o p=1}^{h_{i, j}}\left[\left(1-p_{i s o l, h o p}\right) p_{f, h o p}\right]\right\} \omega_{i, j} / h_{i, j}
$$


Thus:

$$
\begin{aligned}
E\left(C_{X(i)}\right) & =E\left\langle\sum_{j}^{N_{c h}(i)}\left\{\prod_{h o p=1}^{h_{i, j}}\left[\left(1-p_{i s o l, h o p}\right) p_{f, h o p}\right]\right\} \omega_{i, j} / h_{i, j}\right\rangle \\
& =\sum_{j}^{N_{c h}(i)}\left\{\prod_{h o p=1}^{h_{i, j}}\left\{\left[1-E\left(p_{i s o l, h o p}\right)\right] E\left(p_{f, h o p}\right)\right\}\right\} \omega_{i, j} / h_{i, j} \\
& =\sum_{j}^{N_{c h}(i)}\left[\left(1-E\left(p_{i s o l}\right)\right) E\left(p_{f}\right)\right]^{h_{i, j}} \omega_{i, j} / h_{i, j}
\end{aligned}
$$

For multiple sub-channel just provide more QoS with more complexity without more avaiable capability, the capacity formula could be simplified as single channel:

$$
E\left(C_{X(i)}\right)=\left[\left(1-E\left(p_{i s o l}\right)\right) E\left(p_{f}\right)\right]^{h_{i}} \omega / h_{i}
$$

\subsection{The upper bound on capacity for unicast traffics}

Derived from "arbitrary networks" in (Gupta \& Kumar 2000) and formula (23), the upper bound capacity on the ideal unicast traffics happens to be while each node just communicates to the one hop neighbors, $h_{i j}=1$, and has maximum $N / 2$ communication pair, obtain:

$$
E(C)=\sum E\left(C_{X(i)}\right)=\frac{N}{2}\left(1-E\left(p_{i s o l}\right)\right) E\left(p_{f}\right) \omega
$$

And the normalized capacity is:

$$
S=\frac{E(C)}{N \omega}=\frac{1}{2}\left(1-E\left(p_{i s o l}\right)\right) E\left(p_{f}\right)
$$

\subsection{The upper bound on capacity for broadcast traffics}

Case broadcast traffics, in a networks with $N$ nodes, the $N$ nodes received the same message from the same source, thus the average efficiency almost is $\omega / N$ when $N$ is large enough. The upper bound on capacity for broadcast traffic is:

$$
\begin{aligned}
& \arg \max [E(C)]=\arg \max \left[\sum_{i} E\left(C_{X(i)}\right)\right] \\
& =\frac{1}{N} \arg \max \left\{\sum_{i}\left[\left(1-E\left(p_{i s o l}\right)\right) E\left(p_{f}\right)\right]^{h_{i, j}} \omega_{i}\right\}
\end{aligned}
$$

Say $\mathrm{D}$ is the radius of the area covered $\mathrm{WMN}$; define $M=\lceil D / r\rceil$. For simplify analysis, say $\mathrm{D}$ is divided exactly by $\mathrm{r}$, thus $\mathrm{M}=\mathrm{D} / \mathrm{r}$. As in fig 5 , the nodes covering the $\mathrm{k}=0$ circle just needs one hop to the AP; the nodes covering the $\mathrm{k}=1$ ring needs at least two hops. Thus the nodes covering the $\mathrm{k}$ ring, $\mathrm{k}<=\mathrm{M}$, need at least $\mathrm{k}+1$ hops. It is obviously that the number of nodes in the $\mathrm{k}$ ring is: 


$$
N_{k}=(2 k+1)(r / D)^{2} N=(2 k+1) N / M^{2}
$$

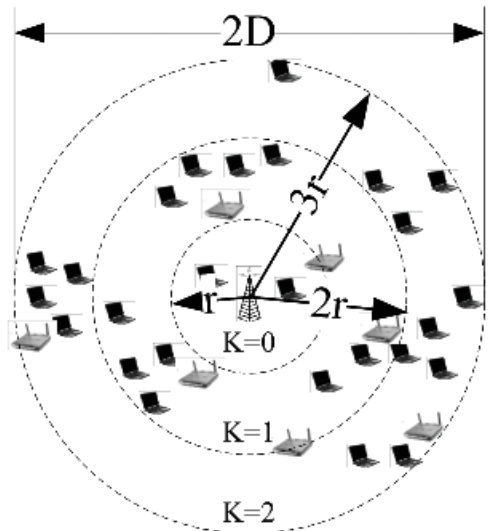

Fig. 5. A scenario for broadcast traffics, case $M=3$

If the number of $\mathrm{AP}$ is 1 ,

$$
\begin{aligned}
\max [E(C)] & =\frac{\omega}{N}\left\{\sum_{k=0}^{M}\left((2 k+1) N / M^{2}\right)\left[\left(1-E\left(p_{\text {isol }}\right)\right) E\left(p_{f}\right)\right]^{k+1}\right\} \\
& =\frac{\omega}{M^{2}}\left\{\sum_{k=0}^{M}(2 k+1)\left[\left(1-E\left(p_{\text {isol }}\right)\right) E\left(p_{f}\right)\right]^{k+1}\right\}
\end{aligned}
$$

And the normalized capacity is

$$
S=\frac{\max [E(C)]}{N \omega}=\frac{1}{N M^{2}}\left\{\sum_{k=0}^{M}(2 k+1)\left[\left(1-E\left(p_{i s o l}\right)\right) E\left(p_{f}\right)\right]^{k+1}\right\}
$$

If there are $N_{A} \mathrm{APs}$, for each $\mathrm{AP}$, similarly get

$$
\begin{gathered}
N_{k, R}=(2 k+1) N / M_{A}{ }^{2} N_{A} \quad, k=0,1,2, \ldots, M_{A} \\
\max [E(C)]=\frac{\omega}{N} \max \left\{N_{A} \sum_{k=0}^{M_{R}} N_{k, R}\left[\left(1-E\left(p_{i s o l}\right)\right) E\left(p_{f}\right)\right]^{k+1}\right\} \\
=\frac{\omega}{M_{A}^{2}} \bullet \max \left\{\sum_{k=0}^{M_{R}}(2 k+1)\left[\left(1-E\left(p_{i s o l}\right)\right) E\left(p_{f}\right)\right]^{k+1}\right\} \\
S=\frac{\max [E(C)]}{N \omega}=\frac{1}{N M_{A}^{2}} \bullet \max \left\{\sum_{k=0}^{M_{R}}(2 k+1)\left[\left(1-E\left(p_{i s o l}\right)\right) E\left(p_{f}\right)\right]^{k+1}\right\}
\end{gathered}
$$

\subsection{The upper bound on capacity of backhual traffics}

For the backhaul traffics, each multi-hop chains has the same capacity $\omega / h$, thus: 


$$
\max [E(C)]=\arg \max \left\{\sum_{i}\left(\sum_{j}^{N_{c h}(i)}\left[\left(1-E\left(p_{i s o l}\right)\right) E\left(p_{f}\right)\right]^{h_{i, j}} \omega_{i, j} / h_{i, j}\right)\right\}
$$

Similar say $M=D / r$ is constant, If there are 1 mesh routers obtaions:

$$
\begin{gathered}
\max [E(C)]=\frac{N \omega}{M^{2}}\left\{\sum_{k=0}^{M}(2 k+1)\left[\left(1-E\left(p_{i s o l}\right)\right) E\left(p_{f}\right)\right]^{k+1} /(k+1)\right\} \\
S=\frac{\max [E(C)]}{N \omega}=\frac{1}{M^{2}}\left\{\sum_{k=0}^{M}(2 k+1)\left[\left(1-E\left(p_{i s o l}\right)\right) E\left(p_{f}\right)\right]^{k+1} /(k+1)\right\}
\end{gathered}
$$

If there are multiple routers:

$$
\begin{gathered}
\max [E(C)]=\frac{\omega N}{M_{A}{ }^{2}} \bullet\left\{\sum_{k=0}^{M_{R}}(2 k+1)\left[\left(1-E\left(p_{\text {isol }}\right)\right) E\left(p_{f}\right)\right]^{k+1} /(k+1)\right\} \\
S=\frac{\max [E(C)]}{N \omega}=\frac{1}{M_{A}^{2}} \bullet\left\{\sum_{k=0}^{M_{R}}(2 k+1)\left[\left(1-E\left(p_{\text {isol }}\right)\right) E\left(p_{f}\right)\right]^{k+1} /(k+1)\right\}
\end{gathered}
$$

\section{Conclusion}

Say $E\left(p_{i s o l}\right)$ is constant,which the density of a networks is cnostant, the capacity on a network is decided by the access probability. With (20), to get the extremum, obstain:

$$
\begin{gathered}
\frac{d\left[E\left(p_{f}\right)\right]}{d v}=-\rho \bullet N \bullet e^{v \bullet \rho \bullet N} \neq 0 \\
\frac{d\left[E\left(p_{f}\right)\right]}{d \rho}=-v \bullet N \bullet e^{-v \bullet \rho \bullet N}+N \bullet e^{-\rho \bullet N} \\
\frac{d\left[E\left(p_{f}\right)\right]}{d N}=-v \bullet \rho \bullet e^{-v \bullet \rho \bullet N}+\rho \bullet e^{-\rho \bullet N}
\end{gathered}
$$

(38) and (39) leads the same conclusion:

$$
\rho \bullet N=\frac{\ln v}{v-1}
$$

While

$$
E\left(p_{f}\right)=e^{-\rho \bullet N}\left(e^{u \bullet \rho \bullet N}-1\right)=e^{-v \cdot \frac{\ln v}{v-1}}-e^{-\frac{\ln v}{v-1}}
$$

The relationship of (40) is shown in fig 6 


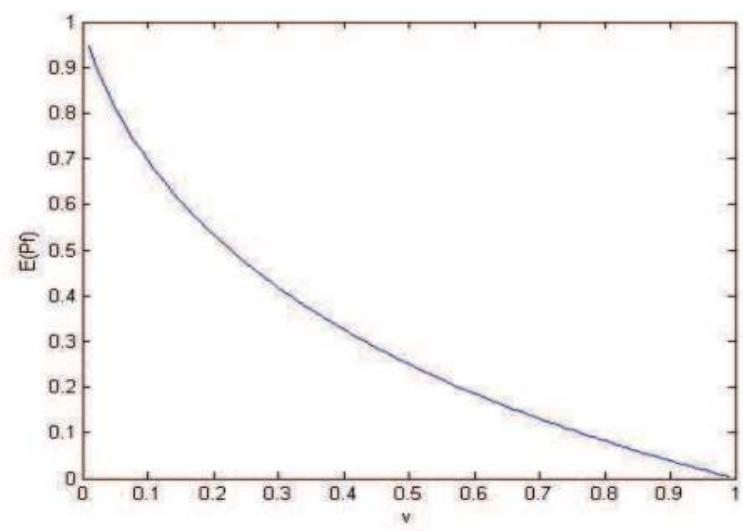

Fig. 6. $E\left(p_{f}\right)-v$ relationship

\section{References}

Akyildiz, I. F. and W. Xudong (2005). "A survey on wireless mesh networks." Communications Magazine, IEEE 43(9): S23-S30.

Basagni, S., D. Turgut, et al. (2001). Mobility-adaptive protocols for managing large ad hoc networks. Communications, 2001. ICC 2001. IEEE International Conference on.

Berrou, C., A. Glavieux, et al. (1993). Near Shannon limit error-correcting coding and decoding: Turbo-codes. . Communications, 1993. ICC 93. Geneva. Technical Program, Conference Record, IEEE International Conference on.

Bharghavan, V., A. Demers, et al. (Aug. 1994). MACAW: A media access protocol for wireless LANs. Proc. SIGCOMM'94 Conf. on Communications Architectures, Protocols and Applications.

Cadambe, V. R. and S. A. Jafar (2007). Degrees of Freedom of Wireless Networks - What a Difference Delay Makes. Signals, Systems and Computers, 2007. ACSSC 2007. Conference Record of the Forty-First Asilomar Conference on.

Cadambe, V. R. and S. A. Jafar (2008). Can feedback, cooperation, relays and full duplex operation increase the degrees of freedom of wireless networks? Information Theory, 2008. ISIT 2008. IEEE International Symposium on.

Cadambe, V. R. and S. A. Jafar (2009). "Degrees of Freedom of Wireless Networks With Relays, Feedback, Cooperation, and Full Duplex Operation." Information Theory, IEEE Transactions on 55(5): 2334-2344.

Chen, Y., G. Zhu, et al. (2008). On the Capacity and Scalability of Wireless Mesh Networks. Wireless Communications, Networking and Mobile Computing, 2008. WiCOM '08. 4 th International Conference on.

Diggavi, S. N., M. Grossglauser, et al. (2005). "Even One-Dimensional Mobility Increases the Capacity of Wireless Networks." Information Theory, IEEE Transactions on 51(11): 3947-3954.

E.Telatar (1999). "Capacity of Multi-Antenna Gaussian Channels." European Transactio on Telecommunications 10(6): 585-595. 
G.J.Foschini (1996). "Layered Space-Tie Architecture for Wirless Communicaton in a Fading Environment when Using Multi-Element Antennas." Bell Labs Technology Journal 1(2): 41-59.

Gallager, R. (1985). "A perspective on multiaccess channels." Information Theory, IEEE Transactions on 31(2): 124-142.

Goldsmith, A. (2005). wireless commnunications, Cambridge University Press.

Grossglauser, M. and D. N. C. Tse (2002). "Mobility increases the capacity of ad hoc wireless networks." Networking, IEEE/ ACM Transactions on 10(4): 477-486.

Gupta, P. and P. R. Kumar (2000). "The capacity of wireless networks." Information Theory, IEEE Transactions on 46(2): 388-404.

Haartsen, J. C. (2000). "The Bluetooth radio system." Personal Communications, IEEE 7(1): 28-36.

Jafar, S. A. (2005). "Too much mobility limits the capacity of wireless ad hoc networks." Information Theory, IEEE Transactions on 51(11): 3954-3965.

Jangeun, J. and M. L. Sichitiu (2003). "The nominal capacity of wireless mesh networks." Wireless Communications, IEEE [see also IEEE Personal Communications] 10(5): 814.

Karn, P. ( Sept.1990). MACA: A new channel access method for packet radio. Proc. 9th Computer Networking Conf.

Khayyat, K. M. J., F. Gebali, et al. (2007). Performance Analysis of the IEEE 802.11 DCF. Signal Processing and Information Technology, 2007 IEEE International Symposium on.

Kumar, P. R. (2003). "A correction to the proof of a lemma in "The capacity of wireless networks"." Information Theory, IEEE Transactions on 49(11): 3117.

Miorando, E. and F. Granelli (2007). On Connectivity and Capacity of Wireless Mesh Networks. Communications, 2007. ICC '07. IEEE International Conference on.

Nandiraju, N., D. Nandiraju, et al. (2007). "Wireless Mesh Networks: Current Challenges and Future Directions of Web-In-The-Sky." Wireless Communications, IEEE [see also IEEE Personal Communications] 14(4): 79-89.

Pham, P. P., P. P. Pham, et al. (2005). Performance Analysis of the IEEE 802.11 DCF. Communications, 2005 Asia-Pacific Conference on.

Samhat, A., A. Samhat, et al. (2006). Performance analysis of the IEEE 802.11 DCF with imperfect radio conditions. Wireless and Mobile Communications, 2006. ICWMC '06. International Conference on.

T.M.Cover and J.A.Thomas (2006). Elements of Information Theory (second edition), John Wiley \& Sons.

Takagi, H. and L. Kleinrock (1984). "Optimal Transmission Ranges for Randomly Distributed Packet Radio Terminals." Communications, IEEE Transactions on [legacy, pre - 1988] 32(3): 246-257.

Tavli, B. (2006). "Broadcast capacity of wireless networks." Communications Letters, IEEE 10(2): 68-69. 


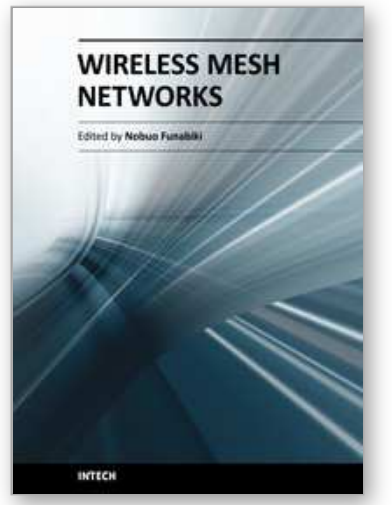

\author{
Wireless Mesh Networks \\ Edited by Nobuo Funabiki
}

ISBN 978-953-307-519-8

Hard cover, 308 pages

Publisher InTech

Published online 14, January, 2011

Published in print edition January, 2011

The rapid advancements of low-cost small-size devices for wireless communications with their international standards and broadband backbone networks using optical fibers accelerate the deployment of wireless networks around the world.â€"The wireless mesh network has emerged as the generalization of the conventional wireless network. However, wireless mesh network has several problems to be solved before being deployed as the fundamental network infrastructure for daily use. The book is edited to specify some problems that come from the disadvantages in wireless mesh network and give their solutions with challenges. The contents of this book consist of two parts: Part I covers the fundamental technical issues in wireless mesh network, and Part II the administrative technical issues in wireless mesh network. This book can be useful as a reference for researchers, engineers, students and educators who have some backgrounds in computer networks, and who have interest in wireless mesh network. It is a collective work of excellent contributions by experts in wireless mesh network.

\title{
How to reference
}

In order to correctly reference this scholarly work, feel free to copy and paste the following:

Yonghui Chen (2011). On the Capacity and Scalability of Wireless Mesh Networks, Wireless Mesh Networks, Nobuo Funabiki (Ed.), ISBN: 978-953-307-519-8, InTech, Available from:

http://www.intechopen.com/books/wireless-mesh-networks/on-the-capacity-and-scalability-of-wireless-meshnetworks

\section{INTECH}

open science | open minds

\section{InTech Europe}

University Campus STeP Ri

Slavka Krautzeka 83/A

51000 Rijeka, Croatia

Phone: +385 (51) 770447

Fax: +385 (51) 686166

www.intechopen.com

\section{InTech China}

Unit 405, Office Block, Hotel Equatorial Shanghai

No.65, Yan An Road (West), Shanghai, 200040, China

中国上海市延安西路65号上海国际贵都大饭店办公楼 405 单元

Phone: +86-21-62489820

Fax: $+86-21-62489821$ 
(C) 2011 The Author(s). Licensee IntechOpen. This chapter is distributed under the terms of the Creative Commons Attribution-NonCommercialShareAlike-3.0 License, which permits use, distribution and reproduction for non-commercial purposes, provided the original is properly cited and derivative works building on this content are distributed under the same license. 